\title{
Robust generalized numerical inspection fixture for the metrology of compliant mechanical parts
}

\author{
Hassan RADVAR-ESFAHLAN ; Souheil-Antoine TAHAN \\ Laboratoire d'ingénierie des produits, procédés et systèmes (LIPPS) \\ École de Technologie Supérieure \\ Montréal, Canada, H3C 1K3
}

\begin{abstract}
Free form nonrigid parts are the inseparable part of today's automotive and aerospace industries. These parts have different shapes in free state due to dimensional and geometric variations, gravity and residual strains. For the geometric inspection of such compliant parts, special inspection fixtures, in combination with coordinate measuring systems (CMM) and/or optical data acquisition devices (scanners) are used. A general procedure to eliminate the use of inspection fixtures was developed by our previous work. Here we apply a new method for robustifying the generalized numerical inspection fixture (GNIF). We filter out points causing incoherent geodesic distances. We demonstrate that our approach has several significant advantages one of which is the ability to handle parts with missing range data. The other advantage of presented method is the capacity to inspect the parts with large deformations.
\end{abstract}

\section{Introduction}

Geometric inspection, geometric modeling, range data acquisition and analysis have developed as separate fields of engineering by different engineering and scientific communities. However, all these fields share a common scientific concept and lack of mutual connections cause the waste in synergy which could be exist. Computer aided inspection is one of these connection points, meanwhile nonrigid geometric inspection share a profound degree of understanding of all mentioned disciplines. As of today, the flexible workpiece has to be fixed or clamped during the measurement process in order to simulate the use state. To this end, special inspection fixture has to be designed and manufactured. On the other hand, some inspection stages cannot be fully automated with this conventional approach. As a result, the geometric inspection of flexible parts is still a time and money consuming process. For example, according to our industrial partner 
(Bombardier Aerospace), some inspection set-up processes for nonrigid parts, demand 60 to 75 hours of operations. This is why the inspection of all manufactured parts in a mass production line is not possible and only statistical control can be performed. Despite the tons of papers and researches on CAD, CAM and CAI (computer aided inspection) fields, inspection of flexible parts poses difficulties and significant cost to industries because they need special fixation devices. This also depicts the lack of knowledge and theoretical foundations around this special field. Our approach [1] was an effort to eliminate the use of special inspection fixtures in metrology of flexible parts. However that method had some drawbacks. Here we depict a clearer picture of it, with regard to the used algorithms. The other aim of this paper is to robustifying the mentioned algorithm. Before giving the details on proposed inspection methodology let's have a definition for some of terms used in this paper.

\section{Definition 1. "Free-state variation is the distortion of a part after removal of forces} applied during manufacture. This distortion is principally due to weight and flexibility of the part and the release of internal stress resulting from fabrication. A part of this kind (e.g. a part with a very thin wall in proportion to its diameter) is referred to as a nonrigid part.” [para. 5.5 ASME Y14.5-2009].

Definition 2. Isometric deformation is a kind of deformations in which the geodesic distance between points preserved during deformation.

Definition 3. Isometric embedding is a distance preserving mapping.

\section{Prior works}

\subsection{Rigid and nonrigid registration}

Parallel to mechanical engineers but in different fields like Computer vision, Biomedical engineering and Pattern recognition, many researches have been done on rigid and nonrigid registration and deformable surface comparison domains. Besl and McKay [2] developed the Iterative closest point (ICP) algorithm an iterative method for the rigid registration of 3D shapes. The ICP algorithm is one of the common techniques for 3D rigid surface registration. Suppose that we are given two shapes $X$ and $Y$. The goal of ICP is to find the rigid transformation which brings two shapes as close as possible. The 
closeness is measured by Hausdorff distance between two shapes. Many versions of ICP have been proposed. They differ from the selection and matching of points, to the minimization strategy [3, 4]. We refer the reader to [5] for an account and comparison between some ICP variants. In [6], Holden provides a comprehensive and quantitative review of spatial transformations models for nonrigid image registration.

Multidimensional scaling (MDS) [7] which widely used and developed in human sciences like sociology and economy was a bridge to represent the intrinsic geometries of the shapes in a common metric space where they can be compared using rigid similarity algorithms. In [8] authors flattened the convoluted surfaces of the human brains in order to compare with each other and finally to study the functional architectures of the brain. For some, their work was a breakthrough in which surface geometry was translated into a plane. From the first generation of MDS until now, many variants of minimization algorithms for minimizing the stress function have been developed. One the most straightforward and successful among those was developed by de Leeuw [9]. In [10] authors presented the concept of Invariant Signature for surfaces. Their method used fast marching on triangulated domains followed by MDS technique. They transformed the problem of isometric-nonrigid surface matching into a matching of rigid surfaces problem. Using MDS, they embedded surfaces $X$ and $Y$ into some common embedding space $Z$ called Canonical form and then measured their similarity using the Hausdorff distance. Their method is strongly based on the Kimmel and Sethian [11] method in computing the geodesic distance on triangular meshes. As the nature of their study was based on classification and pattern recognition, the accuracy of their presented method as a measure of similarity of metric spaces was not discussed. On the other hand the uncertainty of canonical forms as a result of geodesics by means of fast marching method, as well as the uncertainty related to the least square multidimensional scaling process should be investigated in depth. As in our special case the similarity measure plays a critical role, the accuracy of canonical forms in case of nonrigid inspection will be studied using real engineering case studies.

In spite of canonical forms in which two metric space was mapped into some common space, Bronstein et al. [12] proposed a method which mapped two metric shape directly into each other. In spite of classic MDS here the distortion was a kind of similarity 
measure tool. The distortion showed that in which degree two shapes are similar (or dissimilar). Other advantage of their method is the ability to measure the similarity of shapes in which there is no exact nodal correspondence between the sets of range data. The presented method was also suitable in case of partial matching.

\section{$2.2 \quad$ Point cloud segmentation}

In spite of world's continuous phenomena, usually in engineering applications such as image processing and computer vision, we encounter with discrete data. In our application (i.e. geometric inspection) features of range data captured by optic scanners should be extracted. To this end, the edges play an important role as one of the feature detection tools. Various approaches have been proposed to detect edge points in measured range data. Chen and Schmitt [13] represented a method to calculate principal curvatures on triangulated surface. Yang and Lee [14] used surface curvature properties for detection of edge points. They investigated the behaviour of the surface curvature in a cross section of the surfaces: step edge, crease edge, edge formed by a concave/convex surface and a flat surface and finally edge formed by a concave and convex surface. If the range images are relatively noise-free then one can compute first the two principal curvatures, then the zero crossing and extrema of the largest principal curvature.

Alrashdan et al. [15] proposed a hybrid segmentation approach. The edge based segmentation performed by Kohenon network to detect step and roof edge points. They used Laplacian filter and surface normal values at each point as an input of Kohenon network. Then they used mean and Gaussian curvatures in order to perform the region based segmentation. The next step was the integration of the two previous steps.

In $[16,17]$ and more recently [18] authors address the problem of object class segmentation of 3D range data using Markov random field. Although MRF based methods give the acceptable results in object recognition, for feature detection (i.e. finding the boundaries, sharp edges and corners which are the subject of similarity measuring process) this method should be combined by others. On the other hand, the accuracy of MRF based method, in metric space's correspondence measure, should be 
investigated in depth. In this paper, as one of the essential steps of our methodology, a novel method of contour detection will be presented.

\subsection{Geometric metrology of nonrigid parts}

3D geometric inspection of free-forms has become a nonseparable part of automotive and aerospace industries. In spite of the revolution in computer sciences and digital data acquisition devices such as laser scanners, nonrigid shape measurement is strongly based on using the fixation devices to simulate the state of use. Free-form nonrigid geometrical inspection has not well studied and state-of-the-art industries still use fixation devices for this purpose. Rigid registration has been the basis of nonrigid inspection. A state of the art review of the most important measuring techniques is presented in [19, 20] along with their capacity for freeform measuring tasks. Throughout these presented methods [21-24] the manufactured part is assumed to have a similar shape to the CAD model, allowing for comparison. Li et al. [23] used a two-step registration. They used some local properties of surface (e.g. Principal and Gaussian curvature) in order to find the coarse correspondence between CAD-model and range data. Then fine localisation has been done based on least square principal. The benefits of their method in comparison to single step ICP algorithm were not discussed. All presented methods, and most recently Ravishankar, et al. [25], have used rigid registration as similarity measures.

The early efforts for nonrigid inspection were made by Weckenmann and Gabbia [26]. They proposed a measuring method using virtual distortion compensation. The idea behind their method was to deform the actual distorted point cloud into the nominal use state (CAD-model). Feature (e.g. holes and edges) extraction was the key to measure the correspondence between CAD-model and range data. From the acquired point cloud a triangle mesh of the surface was generated. Then the meshed surface was transformed into a FEM model to simulate the fixation process by using extracted features as boundary condition. Their method was not completely automated because the suggested method needed some human challenges to identify the correlation between some special points like holes and assembly joint positions. These led the controller to find the 
boundary conditions of the FEM problematic. Besides, transforming the point cloud to a computer-aided analyzable model is a very time consuming process. These drawbacks then largely improved in [27]. To this end they deformed CAD-model and mapped it towards range data. By this way, they decreased the time of inspection. A FEM-mesh created from a CAD-model, also provided more precise results than a triangle mesh from measurement results. However, proposed method still needed human intervention in order to find the correspondence between CAD-model and range data.

The concept of the Small Displacement Torsor (SDT) was developed by Bourdet and Clément [28] to solve the general problem of a geometrical surface model fit to a set of points using rigid body movements. Lartigue et al. [29] took advantage of the possibilities offered by voxel representation and SDT method for the dimensional metrology of flexible parts. They considered the effect of gravity and the spatial location of a scanned part. This method is fundamentally based on finding the correspondence between the cloud of all measured points and CAD meshed data. In fact, the SDT is not suitable in the presence of large deformations.

Abenhaim et al. [30] developed an Iterative Displacement Inspection (IDI) which smoothly deformed the CAD mesh data until it matched the range data. Their method was based on optimal step nonrigid ICP algorithms [31]. The point cloud needs to be dense enough because the method's similarity measure is based on the nearest distance calculation. The method depends on finding some flexibility parameters which could vary according to thickness. They used the same number of nodes in two datasets which rarely happens in real applications. By the way, they did not test the ICI in presence of topological discontinuities like holes. The mentioned drawbacks cause previously mentioned methods to limit their applicability in industrial applications.

\section{Robust numerical inspection fixture (RNIF)}

In [1] and for the first time in metrology, we presented a methodology based on the fact that the interpoint shortest path (geodesic distance) between any two points on the parts remains unchanged during an isometric deformation. We called this property as distance preserving property of nonrigid parts. Here we provide a more detailed image of it. The aim is to construct a more robust algorithm. To this end we will filter out some geodesic 
distances which influence our algorithm. With depicted examples we will discuss the source of such incoherent geodesics.

\subsection{Geodesics on range data}

Fig. 1 depicts a thin-wall part deformed under its weight. Let $x_{i}$ be the theoretical point within a CAD model and $y_{i}^{\prime}$ represents the image of $x_{i}$ in Y on free-state. Where $x_{1}, \ldots, x_{n} \in X ; y_{1}^{\prime}, \ldots, y_{m}^{\prime} \in Y$ and $n, m$ are the sampled points which represent two spaces $X$ and $Y$. In spite of large deformation, two shapes are intrinsically similar, which means that it is possible to unfold one surface onto the other without stretching it, i.e. a map of one surface onto the other preserving its distance. The shortest path (geodesic distance) between $x_{1}$ and $x_{2}$ rests unchangeable during isometric deformation, so $d_{\overparen{x_{1} x_{2}}}=d_{\overparen{y_{1}^{\prime} y_{2}^{\prime}}}$. As we will discuss later, this property enable us to find the correspondence between CAD model and scanned data. Mathematically speaking intrinsic properties rest unchangeable to isometric deformations; so in order to compare the nonrigid shapes we should look at their intrinsic geometries. By other words, since $\mathrm{X}$ and $\mathrm{Y}$ belong to different metric spaces $\mathbb{R}^{3}$, we cannot measure their similarity using Hausdorff distance based similarity measure (e.g. using ICP).

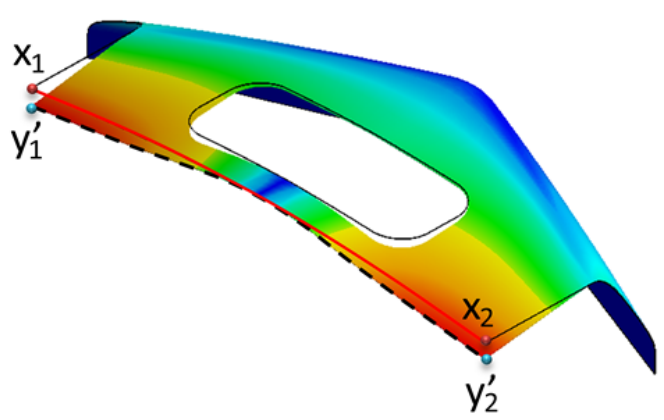

Figure 1

Figure 1: Intrinsic similarity in deformed shapes

Therefore, the first and most important step of our approache is to approximate the pairwise geodesic distance between all points on range data and CAD model. If the sampling domain is dense enough, one idea is to approximate the geodesic distance 
between points with the famous Dijkstra's shortest path algorithm [32]; but the shortest path computed by Dijkstra algorithm does not always lead to the real shortest path. As an example consider a unit square graph and its shortest path between upper left and lower right which is equal to $\sqrt{2}$ while the shortest calculated by Dijkstra algorithm is equal to 2. This problem is due to the fact that we are allowed to move in the graph using only nodal points. To overcome to this inconsistency of Dijksta algorithm Fast Marching Method (FMM) was introduced by Sethian [33] as a numerical method for solving boundary value problems of the Eikonal equation:

$$
|\nabla T| F=1, \quad T\left(X_{0}\right)=0
$$

which describes the propagation of a closed curve in $\mathbb{R}^{2}$ (or a surface in $\mathbb{R}^{3}$ ) with speed $\mathrm{F}$ in the direction normal to itself so that the sign of speed function never change. Kimmel and Sethian [11] have developed a version of Fast Marching algorithm on triangulated domains with the same computational complexity. The initialization of $T$ is like in Dijkstra algorithm, zero at $X_{0}$. Unlike the Dijksta algorithm which the shortest path was restricted to graph vertices, in FMM the shortest path can pass through the triangular mesh. Fig. 2 (left) depicts the idea behind FMM in triangulated domain. Suppose that we are given two points $x_{1}, x_{2}$ with known front arrival times $\mathrm{T}_{1}$ and $\mathrm{T}_{2}$. The question is how to estimate the front time $T_{3}$ when it arrives to the point $x_{3}$. Note that we can freely switch between the path length and arrival time, thus $d_{1}=\mathrm{T}_{1}$, etc. In this case $d$ can be calculated as point-plane distance $d_{1}=n \cdot x_{1}+p, d_{2}=n \cdot x_{2}+p$. These two equations can be written with matrix notation:

$$
V^{T} n+p \cdot 1=d
$$

where $d=\left[d_{1}, d_{2}\right]^{\mathrm{T}}, \mathrm{V}=\left[x_{1}, x_{2}\right], 1=[1,1]^{\mathrm{T}}$. Unit normal vector $n$ can be easily driven from equation (2). 

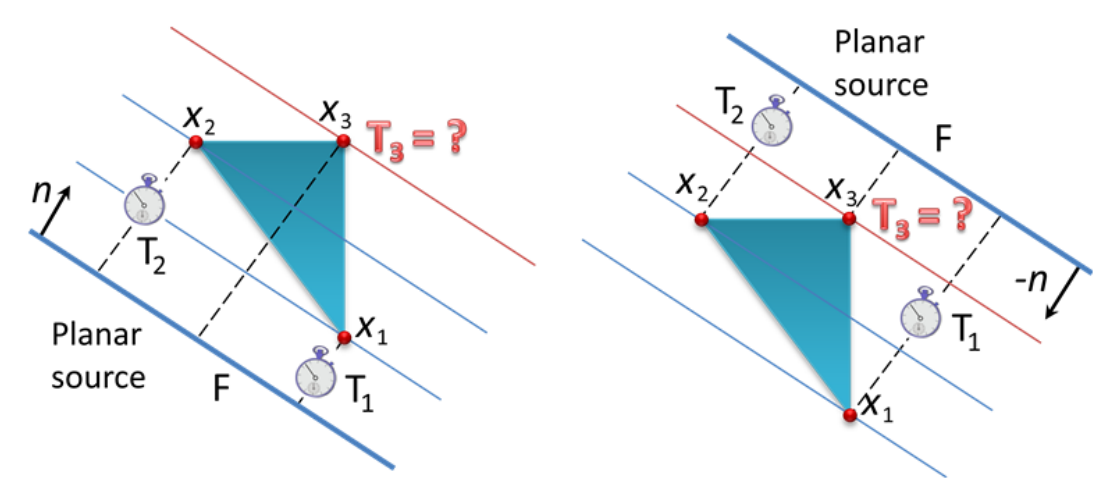

Figure 2 : Calculation of arrival time in $\mathbb{R}^{2}$ for a expanding front $F>0$.

Assuming that the front is planar $\left(x_{1}, x_{2}, x_{3} \in \mathbb{R}^{2}\right)$, the discretization of the Eikonal equation leads to the following quadratic equation [34]:

$$
d_{3}^{2} \cdot 1^{T} Q 1-2 d_{3} \cdot 1^{T} Q d+d^{T} Q d-1=0
$$

where $\mathrm{Q}=\left(\mathrm{V}^{\mathrm{T}} \mathrm{V}\right)^{-1}$. Equation (3) has two solutions, both $n$, $-n$ satisfy the equation. In this case the smallest solution is not acceptable (Fig. 2 right) because front propagation time is monotonically increasing function which means that $d_{3}>d_{1}, d_{2}$. This is equal to say that $V^{T} n<0$.

Other effect of this monotonicity can be translated as:

$$
\left(\frac{\partial d_{3}}{\partial d_{1}}, \frac{\partial d_{3}}{\partial d_{2}}\right)^{T}>0
$$

which means that $d_{3}$ should increased when $d_{1}, d_{2}$ increase or simply $Q V^{T} n<0$. Seeing that $\mathrm{Q}=\left(\mathrm{V}^{\mathrm{T}} \mathrm{V}\right)^{-1}$ then $Q V^{T} V=\left(V^{T} V\right)^{-1} V^{T} V=I$. This means that rows of $Q V^{T}$ are orthogonal to triangle edges which mean that $n$ must lie within triangle and $\measuredangle x_{1} x_{3} x_{2}$ should be acute. Thus, the entire update step can be summarized as in Algorithm 1. 


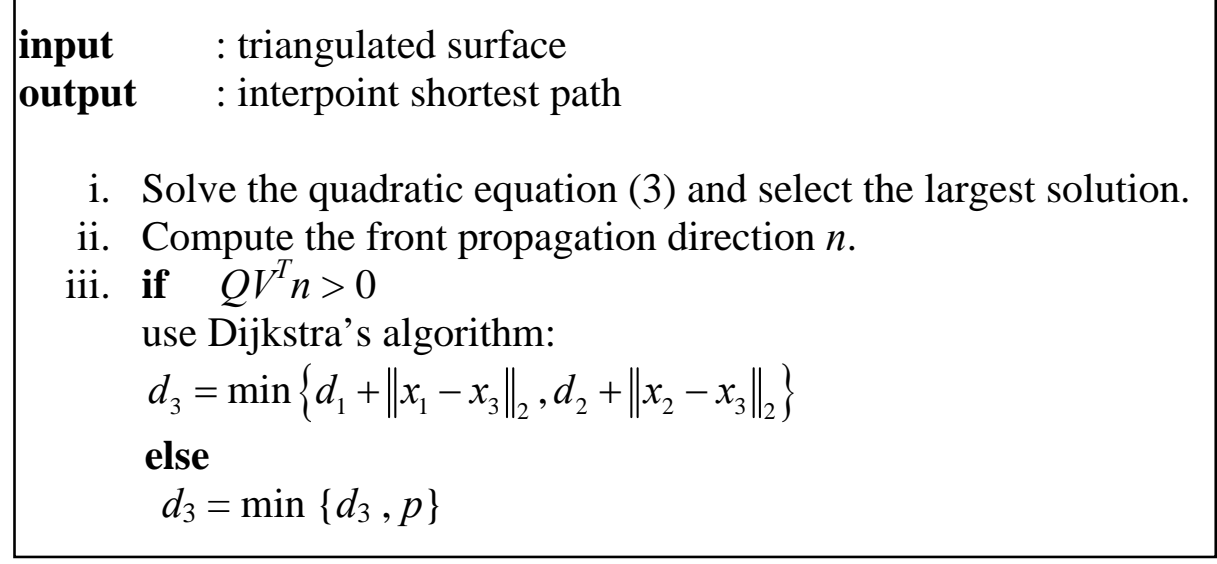

Algorithm 1: Fast marching update

In case of obtuse meshes in [11] the authors propose to split obtuse triangle by into two acute ones.

\subsection{Isometric embedding}

Let $X$ and $Y$ be metric spaces and $f: X \rightarrow Y$ an arbitrary map. The distortion of $f$ is defined by:

$$
\operatorname{dis} f=\sup _{a . b \in X}\left|d_{Y}(f(a), f(b))-d_{X}(a, b)\right|
$$

The distance $d_{X}(a, b)$ between a pair of points in $X$ is mapped to the distance $d_{Y}(f(a), f(b))$ between the images of $a$ and $b$ under $f$ [35]. 


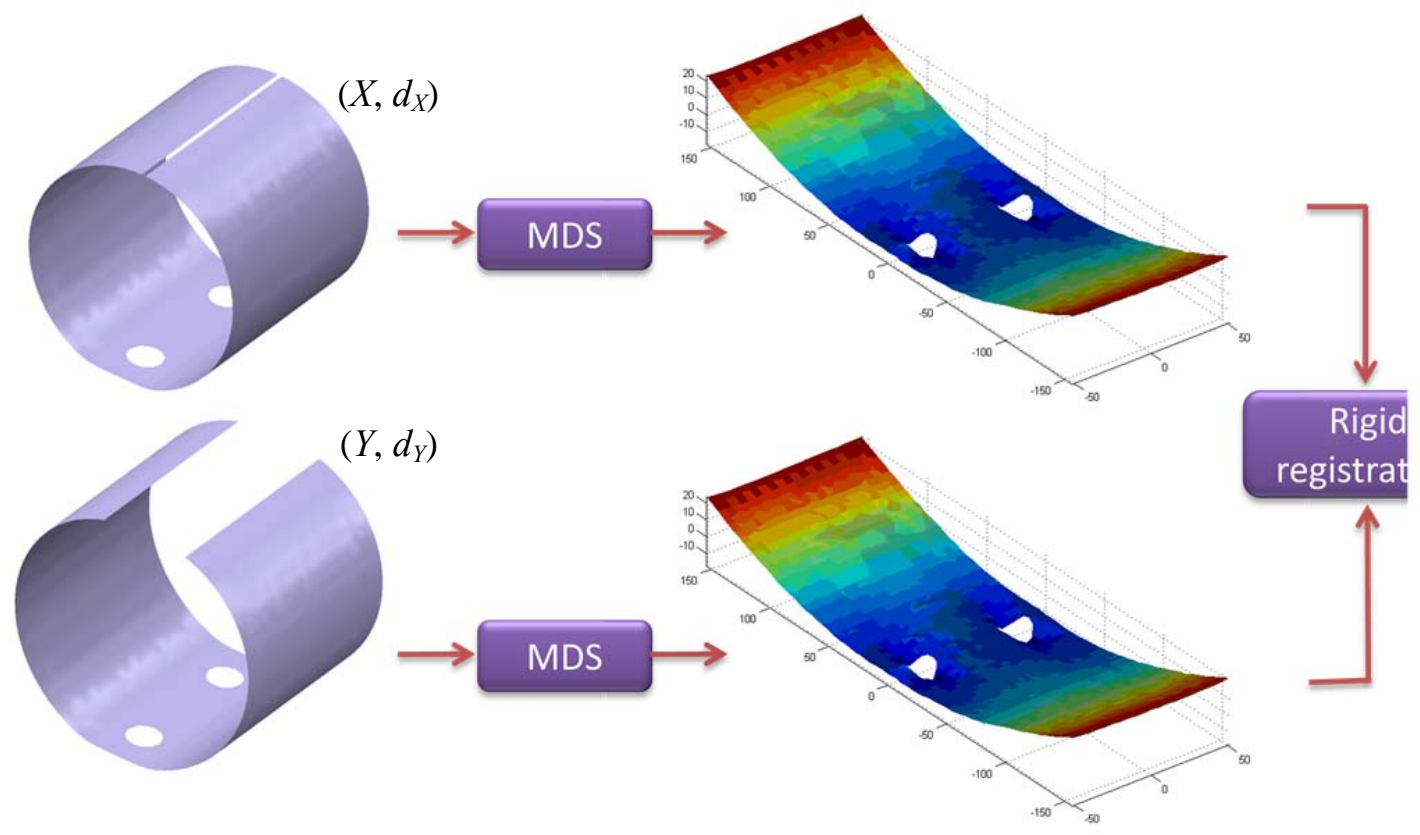

Figure 3 : Illustration of the isometric embedding problem.

Fig. 3 depicts two similar nonrigid shapes. As stated in the previous section our goal is to compare and find the correspondence between shapes $X$ and $Y$ with the metrics of $d_{X}$ and $d_{Y}$. In most of applications for nonrigid parts the deformations are isometric. This means that two shapes $X$ and $Y$ in spite of deformations- are isometrically equal. In this case, because two metrics are different we cannot find their similarity using Hausdorff based methods like ICP. In fact nonrigid registration is somehow difficult from that of the rigid registration. In spite of rigid case, here we should look for the intrinsic similarity because intrinsic geometry remains unchangeable during isometric deformations. Fig. 3 also demonstrates a method in order to deal with nonrigid registration problem. Suppose that we are capable to embed two shapes (left) in a common metric space (right). If we can construct this embedding in such way that the Euclidean distance between all interpoint nodes during embedding remains equal as for geodesics before embedding, in this case we can transform nonrigid similarity problem into a rigid registration problem. This method seems like an ideal method for nonrigid registration problem. The only problem is how to map the shapes into common space in an ideally isometric manner. From our knowledge of geometry we know, for example, that mapping a sphere into flat surface 
(and vise versa) produces unwanted distortions; because each of them has a different Gaussian curvature. A way to overcome such a problem is to try to construct an approximate construction by minimizing the distortion as defined in equation (5). This is the basic idea behind the canonical forms proposed in [10]. In our point cloud setting, where the shape $X$ is sampeled at $N$ points $\left\{x_{1}, x_{2}, \ldots, x_{N}\right\}$, the distortion criteria will be:

$$
\sigma=\max _{i, j=1, \ldots, N}\left|d_{\mathbb{R}^{m}}\left(f\left(x_{i}\right), f\left(x_{j}\right)\right)-d_{X}\left(x_{i}, x_{j}\right)\right|
$$

In MDS literature the function $\sigma$ which measures the distortion of distances is called stress. Historically $\sigma_{2}$ is used as the distortion criterion. Assume that $Z_{i}=f\left(x_{i}\right)$ is a matrix of canonical form coordinates and $d_{i j}(Z)=d_{\mathbb{R}^{m}}\left(z_{i}, z_{j}\right)$, then:

$$
\sigma_{2}\left(Z ; D_{X}\right)=\sum_{i>j}\left|d_{i j}(Z)-d_{X}\left(x_{i}, x_{j}\right)\right|^{2}
$$

Here $D_{X}$ is a matrix of geodesic distances and $d_{i j}(Z)$ is the Euclidean distance between the points on the canonical form. The minimization algorithms which minimize the stress function known as Multidimensional scaling. Historically MDS is classfied as a dimensionality reduction method. SMACOF (scaling by majorizing a complicated function) is one of the well known MDS algorithms for minimizing the stress function $\sigma_{2}\left(Z ; D_{X}\right)$ with respect to $Z$. This algorithm was proposed by proposed by De Leeuw [9]. This algorithm is the core of our study in [36]. Here we present a brief introduction on SMACOF. We refer the reader to [7] for an account. Before summarizing the SMACOF algorithm we describe some relations and notations. Equation (7) can be written as matrix form:

$$
\sigma_{2}\left(Z ; D_{X}\right)=\operatorname{tr}\left(Z^{T} V Z\right)-2 \operatorname{tr}\left(Z^{T} B\left(Z ; D_{X}\right) Z\right)+\sum_{i>j} d_{X}^{2}\left(x_{i}, x_{j}\right)
$$

Here $V$ is a constant $N \times N$ matrix with elements:

$$
v_{i j}=\left\{\begin{array}{cc}
-1 & i \neq j \\
N-1 & i=j
\end{array}\right.
$$

and $B\left(Z ; D_{X}\right)$ is an $N \times N$ matrix with elements: 


$$
b_{i j}\left(Z ; D_{X}\right)=\left\{\begin{array}{ccc}
-d_{X}\left(x_{i}, x_{j}\right) d_{i j}^{-1}(Z) & i \neq j \quad \text { and } d_{i j}(Z) \neq 0 \\
0 & i \neq j \quad \text { and } d_{i j}(Z)=0 \\
-\sum_{i \neq k} b_{i k} & i=j &
\end{array}\right.
$$

Thus, the SMACOF algorithm can be summarized as:

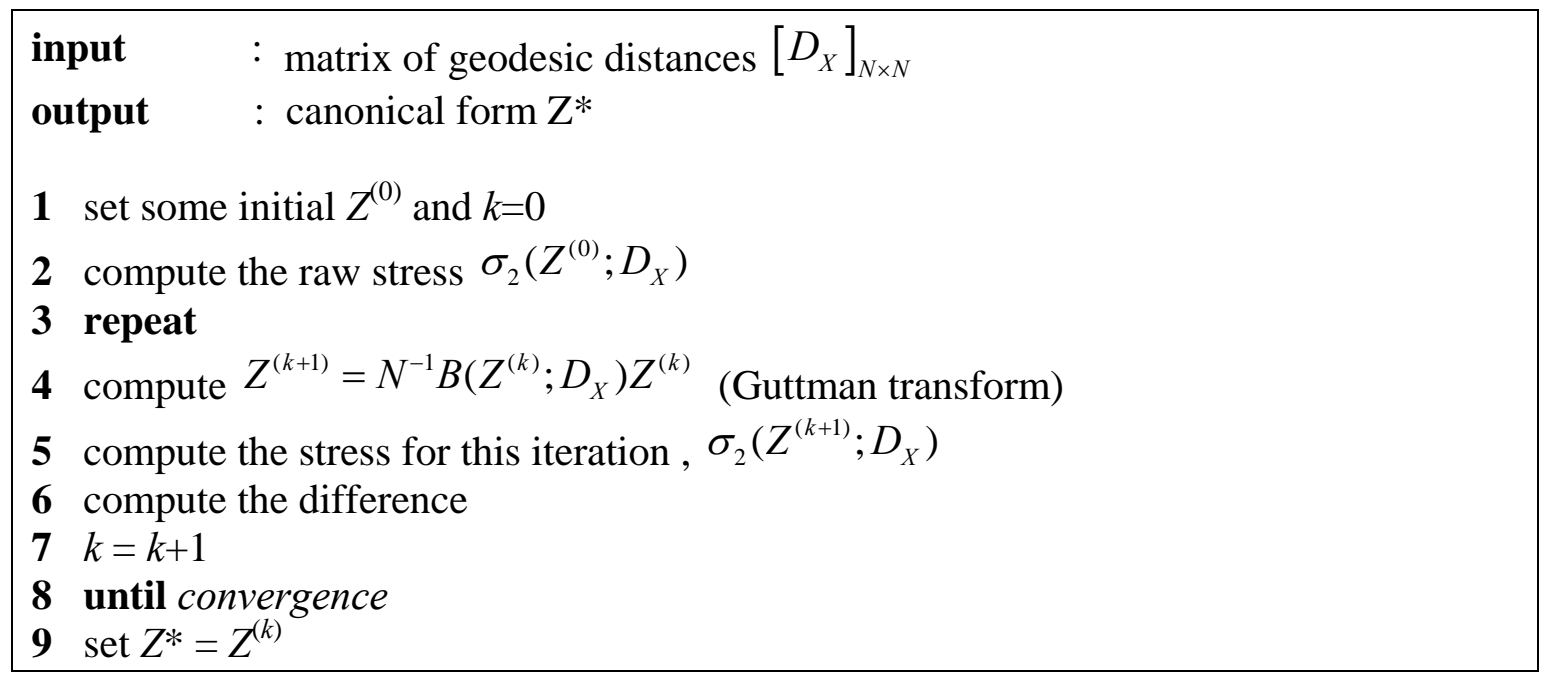

Algorithm 2 : SMACOF algorithm

Step 6 of SMACOF algorithm contains of finding the difference in the stress values between the two previous iterations. If it is less than some predefined tolerance or if the maximum number of iterations has been reached, then algorithm will stop. Despite of simplicity, SMACOF guarantees monotonically decreasing sequence of stress values. Fig. 4 illustrates the convergence of SMACOF algorithm applied to the problem of embedding of surface $X$ (Fig. 3) sampled with $N=1511$ points. 


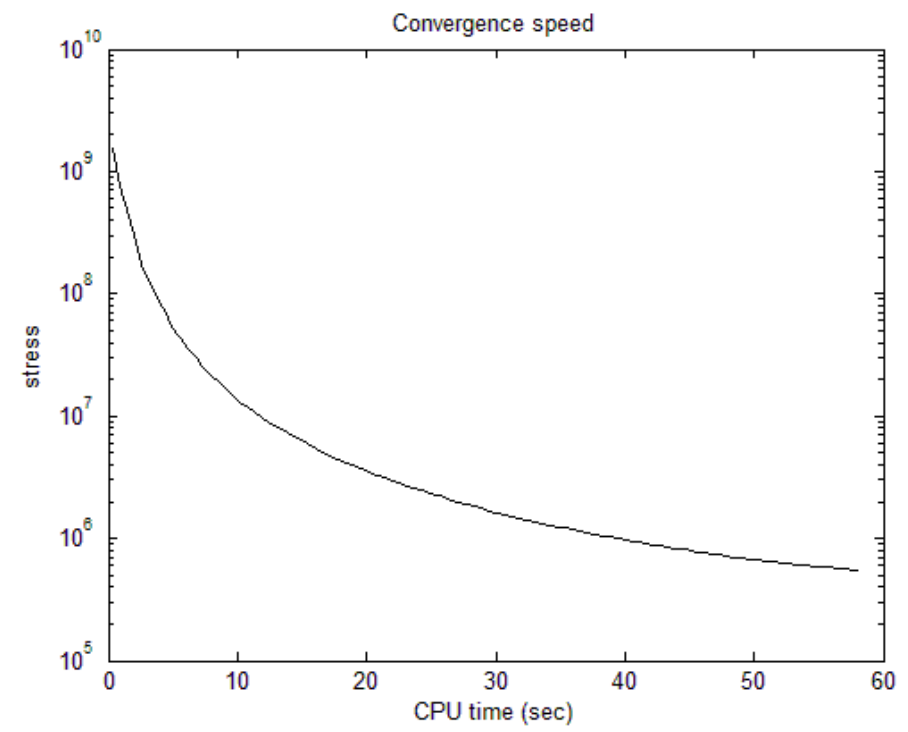

Figure 4: convergence plot of SMACOF algorithm applied to shape $X$ (Fig. 3) with $\mathrm{N}=1511$ nodes.

\subsubsection{Generalized multidimensional scaling}

In the previous section we saw that MDS always looks for Euclidean embedding space.

We also discussed that such an Euclidean embedding is rarely without distortion especially if one try to embed a surface that looks more like sphere than a plane into Euclidean space. One way to overcome this drawback is by choosing one of the surfaces, say $Y$ as embedding space (Fig. 5). In other words, we would like to embed $\mathrm{X}$ into $\mathrm{Y}$ by solving the following problem:

$$
\min _{y_{1}^{\prime}, \ldots, y_{N}^{\prime} \in Y} \sum\left|d_{X}\left(x_{i}, x_{j}\right)-d_{Y}\left(y_{i}^{\prime}, y_{j}^{\prime}{ }_{j}\right)\right|^{2}
$$

where $y_{i}^{\prime}$ is the image of $x_{i}$ in $Y$. 

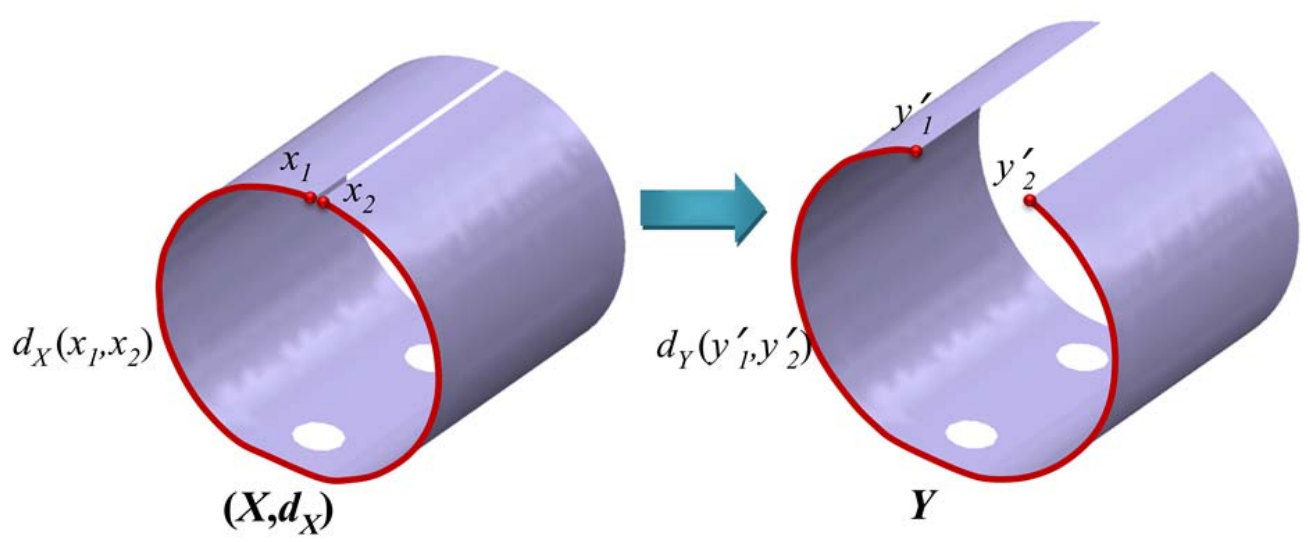

Figure 5: Generalized MDS

In this case, the minimum stress value quantifies how much the metric of $X$ should be distorted in order to fit into $Y$. But unlike MDS, there is no closed-form expression for $d_{Y}\left(y_{i}^{\prime}, y_{j}^{\prime}\right)$ and the metric needs to be approximated, as $y_{i}^{\prime}$ are the optimization variables. Simply speaking, matrix of pair-wise geodesic distances $d_{X}\left(x_{i}, x_{j}\right)$ can be precomputed, since $\left\{x_{i}\right\}$ are fixed but the question is how to compute $d_{Y}\left(y_{i}^{\prime}, y_{j}^{\prime}\right)$. Assuming two arbitrary points on the meshed surface $y_{i}=\left(t_{i}, u_{i}\right)$ and $y_{i}^{\prime}=\left(t_{i}^{\prime}, u_{i}^{\prime}\right)$ in barycentric coordinates (any point inside a triangle can be expressed uniquely as a convex combination of the triangle vertices) the goal is to calculate the geodesic distance between $y_{i}$ and $y_{i}^{\prime}$.To this end, in [34] and, the authors represent the three point geodesic distance interpolation. They proved that:

$$
\hat{d}_{Y}\left(y, y^{\prime}\right)=u^{\prime T} D_{Y}\left(t, t^{\prime}\right) u
$$

in which, the matrix $D_{Y}\left(t, t^{\prime}\right)$ only depends on triangle indices $t$ and $t^{\prime}$. Substituting the interpolated distance term (11) into generalized stress function one can obtain,

$$
\sigma\left(t_{1}, u_{1}, \ldots, t_{N}, u_{N}\right)=\sum\left(d_{X}\left(x_{i}, x_{j}\right)-u_{i}^{T} D_{Y}\left(t_{i}, t_{j}\right) u_{j}\right)^{2}
$$

Fixing all $u_{j}$ and all $t_{j}$, the stress as a function of $u_{i}$ becomes quadratic. Thus, the minimization algorithm can be summarized by: 


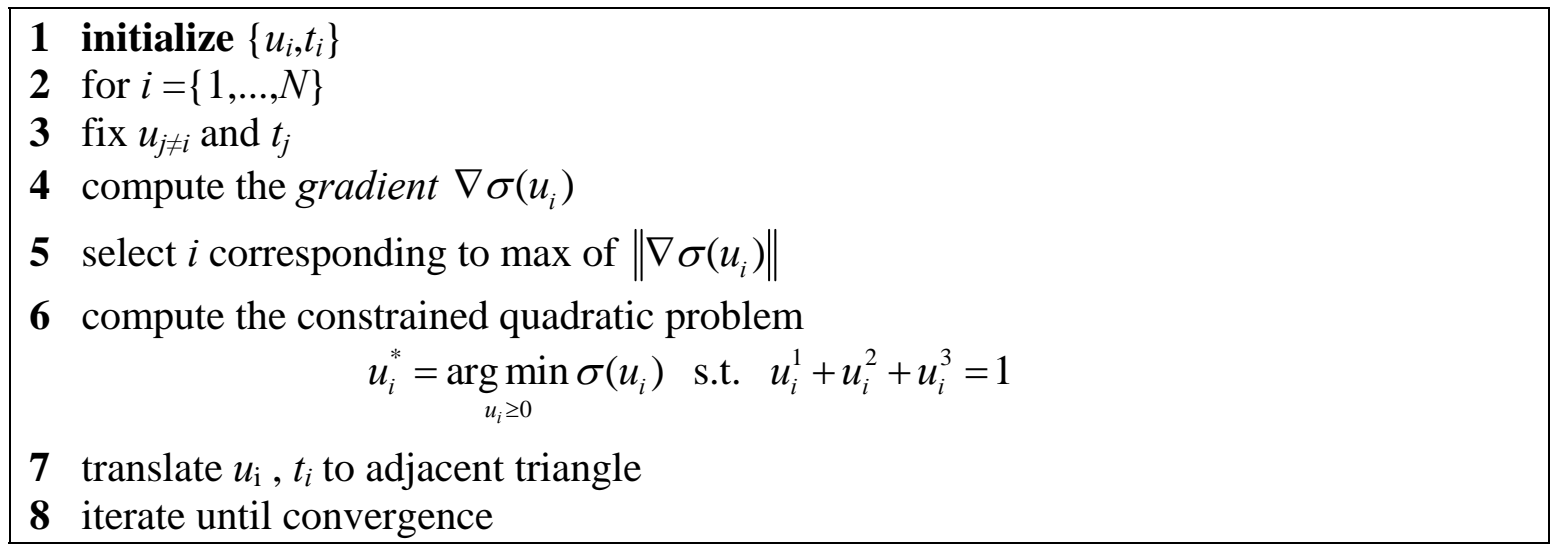

Algorithm 3 : Least square GMDS algorithm

Algorithm 3 is not the only way to solve the generalized MDS problem. We refer the reader to [37] for further account.

\subsection{Incoherent geodesic distances}

In this section we are going to discuss about what we call it incoherent geodesics and the origin of incoherency. Fig. 6a and 6b depict $X$ and $Y$ as two metric spaces corresponding to CAD-model and range data. In Fig. 6a and at the middle of the part (white) consider an area with missing data. This is normally a common problem during data acquisition process. Missed data can be caused by operator's error or scanners precision. Data sampling and meshing also can cause such a missed data region. While the actual geodesic between $x_{i}$ and $x_{j}$ in CAD model is calculated by straight (black) line, the geodesic in range data (red dashed line) significantly varies from nominal one (Fig. 6a). Fig. $6 \mathrm{~b}$ depicts a case in which the operator decides to do a partial inspection. In this case also the actual geodesic (red line) in boundary of range data may vary from nominal one (black line - in CAD model).

In this study we will use the boundary of the parts in order to perform the nonrigid finite element registration. This will be discussed in section 3.4. In most cases the boundary of the parts is mostly contaminated area with noise and geometric deviation. Thus, it may be another source of incoherency. So far we know two kinds of these geodesics: 1) ones who have the contact with missing data region, 2) others who have a contact with boundary of 
the scanned part. We will filter all these geodesics which we call them incoherent geodesics out of MDS procedure.

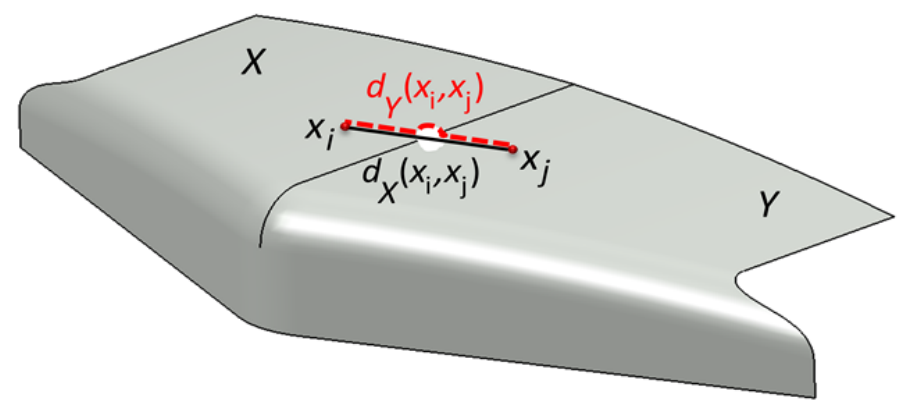

(a)

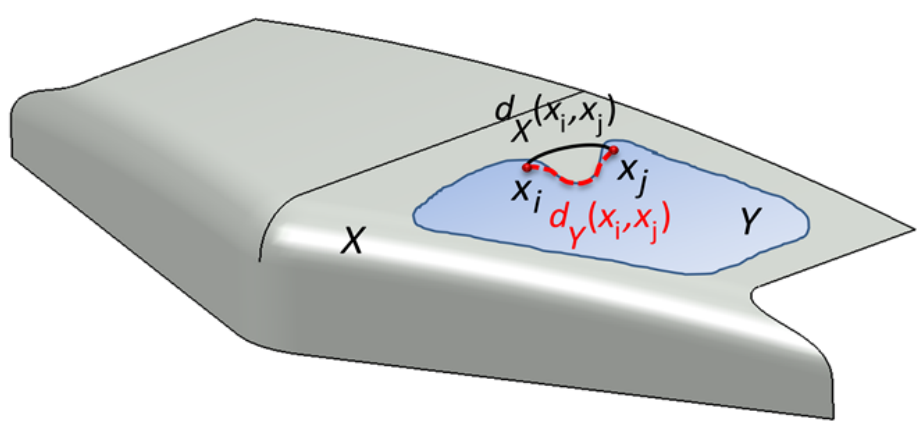

(b)

Figure 6: Incoherency caused by missing data (a); and partial matching (b)

\subsubsection{Boundary detection}

Geodesic distances are vital and critical as the inputs for MDS algorithm. Any incoherency can cause the inaccurate correspondence between CAD-model and range data. To prevent such situation, we find all geodesics which have a contact with the mentioned regions and filter points causing such an incoherent geodesic distance. To this end, we propose a simple method to calculate the boundaries. Of course that there exist many algorithms for edge and boundary detection in range data (section 2.2) our 
method's simplicity comes from the ability of transforming the higher dimensional data into lower dimensional space.

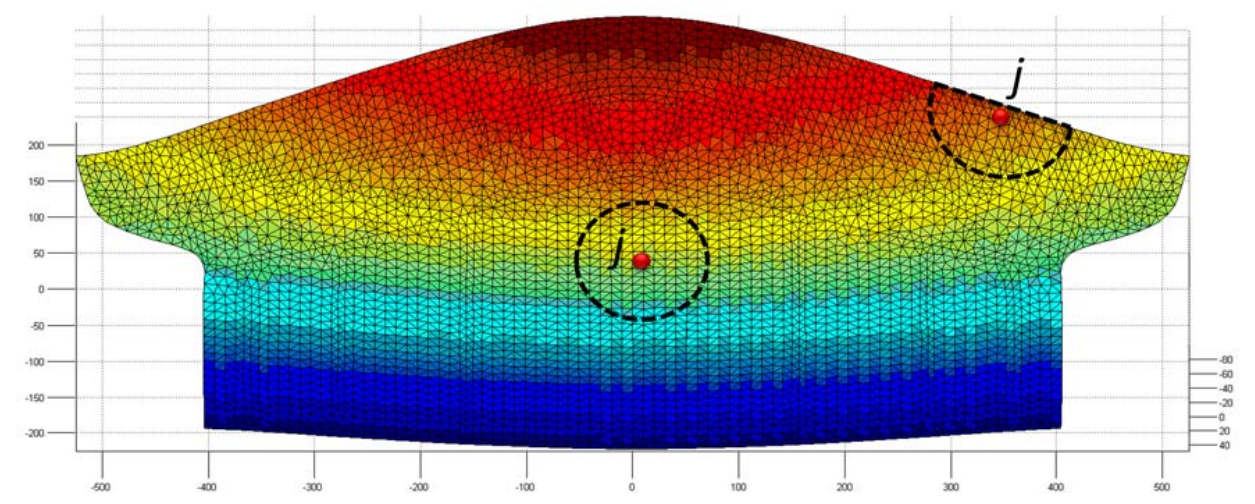

Figure 7: Boundary detection for the part in Fig. 6 (embedding using SMACOF)

In Fig. 7 any point $j$ is a boundary point if all its neighbours are on one side of it. This simple idea leads us into following boundary detection algorithm.

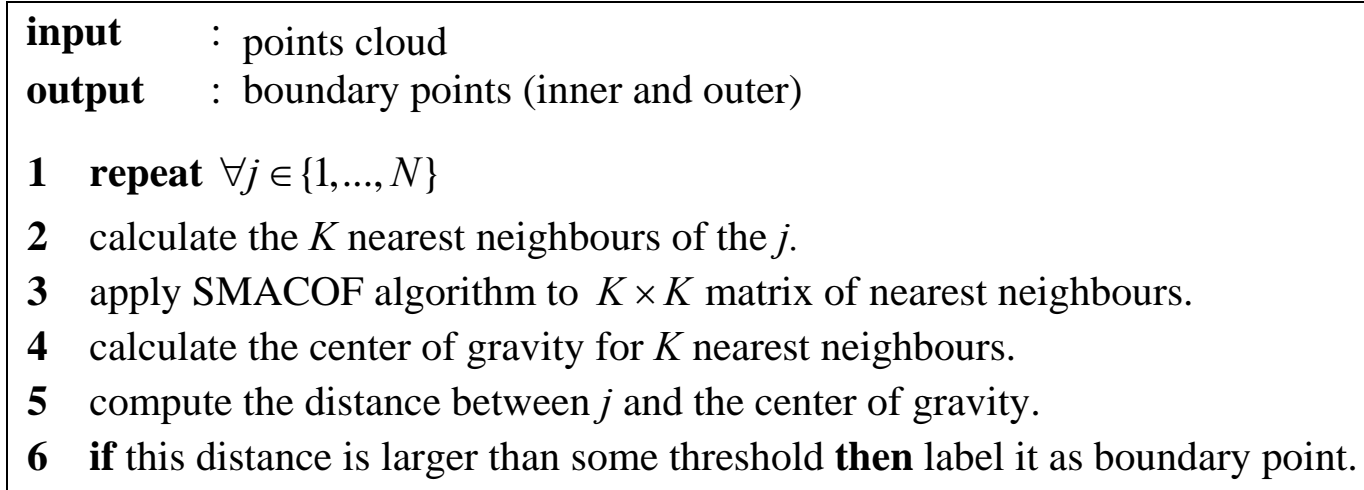

Algorithm 4 : Boundary detection algorithm

\subsection{Nonrigid finite element registration}

Our methodology was inspired by real industrial inspection process. When we put the flexible part on the inspection fixture the prevailing idea is that we are going to simulate the state of use. This is absolutely correct! But more specifically we can say that we are looking for some correspondence between distorted part and inspection fixture, which represents our CAD-model. We present a methodology based on the fact that the 
interpoint shortest path (geodesic distance) between any two points on a shape remains unchanged during an isometric deformation. We call this property as distance preserving property of nonrigid parts. In Fig. 8, CAD-model and range data are represented as a cantilever beam. For simplicity and without loss of generality, let's assume that some of prior information about boundary conditions is already known (e.g. support point). Rigid registration (e.g., ICP based algorithm) can be done using this prior information. In the absence of plastic deformations, displacing $x_{1}$ to $y_{1}$ will deform the beam; so that there will be a bijective (one-to-one correspondence) distance-preserving map between these two shapes (by bijective we do not mean the exact nodal correspondence). Also we assume that all pair-wise geodesic distances between the points on $X$ (CAD-model) and $Y$ (scanned data) are available (e.g., using fast marching method). If we can introduce similarity measure in order to find correspondence between these two metric spaces, the step that we call it finite element nonrigid registration (FENR) can be performed: (a) Find the correspondence (e.g. $y_{1}$ is the image of $x_{1}$ ). (b) Knowing some boundary conditions as prior information and finding the correspondence then displace $x_{1}$ towards $y_{1}$. (c) Calculate the geometric deviation between deformed CAD-model and measure range data.
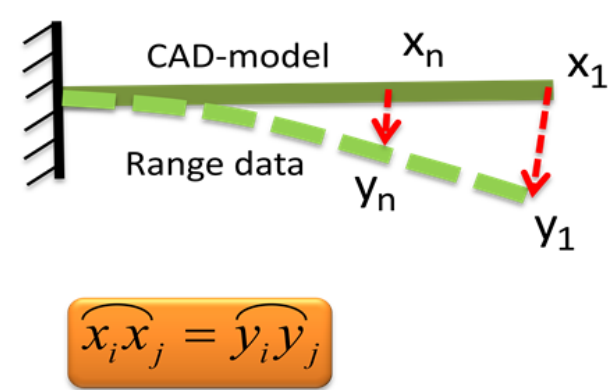

"Distance preserving property of nonrigid parts"

Figure 8: Nonrigid finite element registration 


\subsection{Identification of geometric deviation}

The part is setup onto reference support points where the position is clearly defined within the part frame. These points, as priori information, will be utilized as the boundary condition, where it will simulate the gravity and support effect on the CAD-model. The part is scanned in a distorted state without a fixation device. Preprocessed measured data then comes together with the CAD-model. Note that the CAD-model should be previously analyzed, applying the gravity and support effects in the same direction as the scanning process (Fig. 9). The transformations that map the preprocessed CAD-model towards range data can be obtained by a regular ICP method. In practice and at this stage, we put the measuring part on the inspection fixture. In our methodology, this range data plays the role of inspection fixture and we call it numerical inspection fixture. Again we notice that mapping the CAD-model into range data has some advantages. Transforming the range data into a computer-aided analyzable model especially for complicated surfaces is a very time consuming process. For such surfaces, more human intervention is needed. Furthermore, parts with hidden stiffening structure or other details at the back side of scanned surface, are so difficult to be modelized as a FEM-analyzable model. The main advantage of proposed method is that only one FEM-analyzable model should be created. This really decreases inspection time specially is mass production inspection. Using geodesic distance as a similarity measure tool, enable us to find the correspondence between CAD-model and range data even in the presence of large deformations. Also note that embedding process does not need primary surface registration, so the similarity detection can take place before the rigid registration. If we suppose that there is no a priori information for the assembly process, then the contour or other assured points with negligible deformations such as rigid attachments can be used for nonrigid mapping of preprocessed CAD-model into the range data. Generalized multidimensional scaling can be used as isometry-invariant partial surface matching so there is no need for perfect contour hypothesis. This is very useful when dealing with parts containing missing data. Defects due to geometric deviation can be found after finite element nonrigid registration, eliminating the spring-back effect. Also note that the meshed CAD-model and the scanned workpiece may have a different number of vertices. 


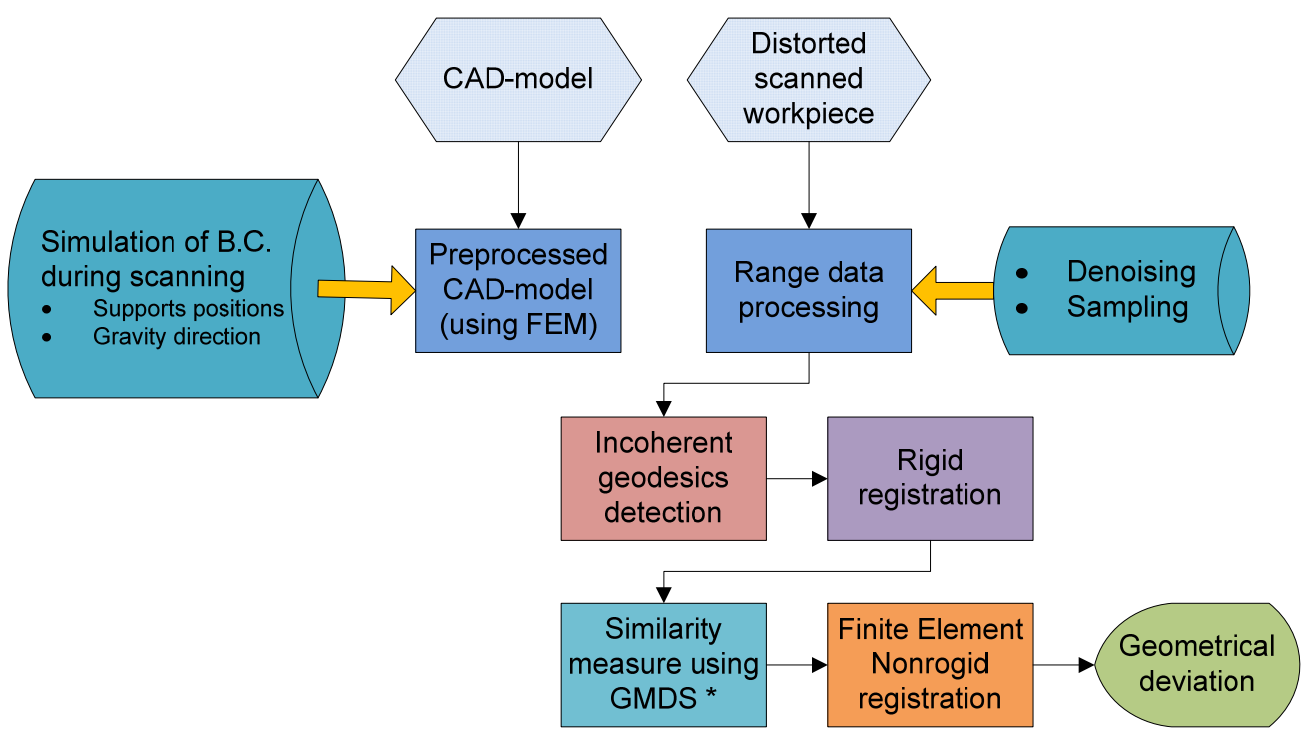

Figure 9: Inspection process flowchart using Robust GNIF

*Similarity measure can also be performed before rigid registration.

The part can be scanned in a production line or it is indispensable to the fast scanning process. The other steps are realized offline using a PC, thus there is no more need to stop production lines for testing a part.

\section{Results}

We have tested presented methodology with a series of typical mechanical parts. Table 1 presents the overall size and engineering data for these parts. We present four case studies containing free-forms, sharp edges, discontinuities (holes) and with different sizes that evaluates performance and validates the method developed in previous section. To this end, the free-form model was simulated by CATIA ${ }^{\circledR}$ then a finite element analysis of the model performed simulating the free-state range data. At this step, a displacement and/or a force was applied to the model to simulate unknown spring back deformation. Fig. 10 depicts such free-state simulation for first case study. Predefined profile defect was also added to all case studies. For instance Fig. 11 illustrates such profile defect for only one blade of propeller. 
Due to the fact that we have used predefined deformation in range data generation steps (spring back and profile defect), qualitative performance evaluation is effectively traceable. Point clouds of free-form and free-state are simulated with a different number of vertices to evaluate the geodesic distance interpolation. We have used Voronoi tessellation in order to represent the sampled discrete nodes of (metric) surface. Then incoherent geodesic detection was performed. To this end we filtered out all the geodesics containing the inner and outer boundary points. In each part we also added the regions with some missing data. After ICP based rigid registration, similarity detection has been done using GMDS. Then CAD-model was mapped towards range data using detected points. Finally the finite element nonrigid registration was performed. Here, only the maximum geometric deviation is presented (Table 2). For better visualization, a sampled tessellated section of the first case study with 50 sampled points is illustrated in Fig. 12. Geodesic distance interpolation enables us to accurately measure the similarity between the $\mathrm{CAD}$ and scanned data; still there is no exact nodal correspondence. In rigid registration process, some prior defined points, or in the areas with the least defect probability, may be used for increasing the procedure speed. The results are shown in Table 2. 
Table 1: Case study's size and engineering data

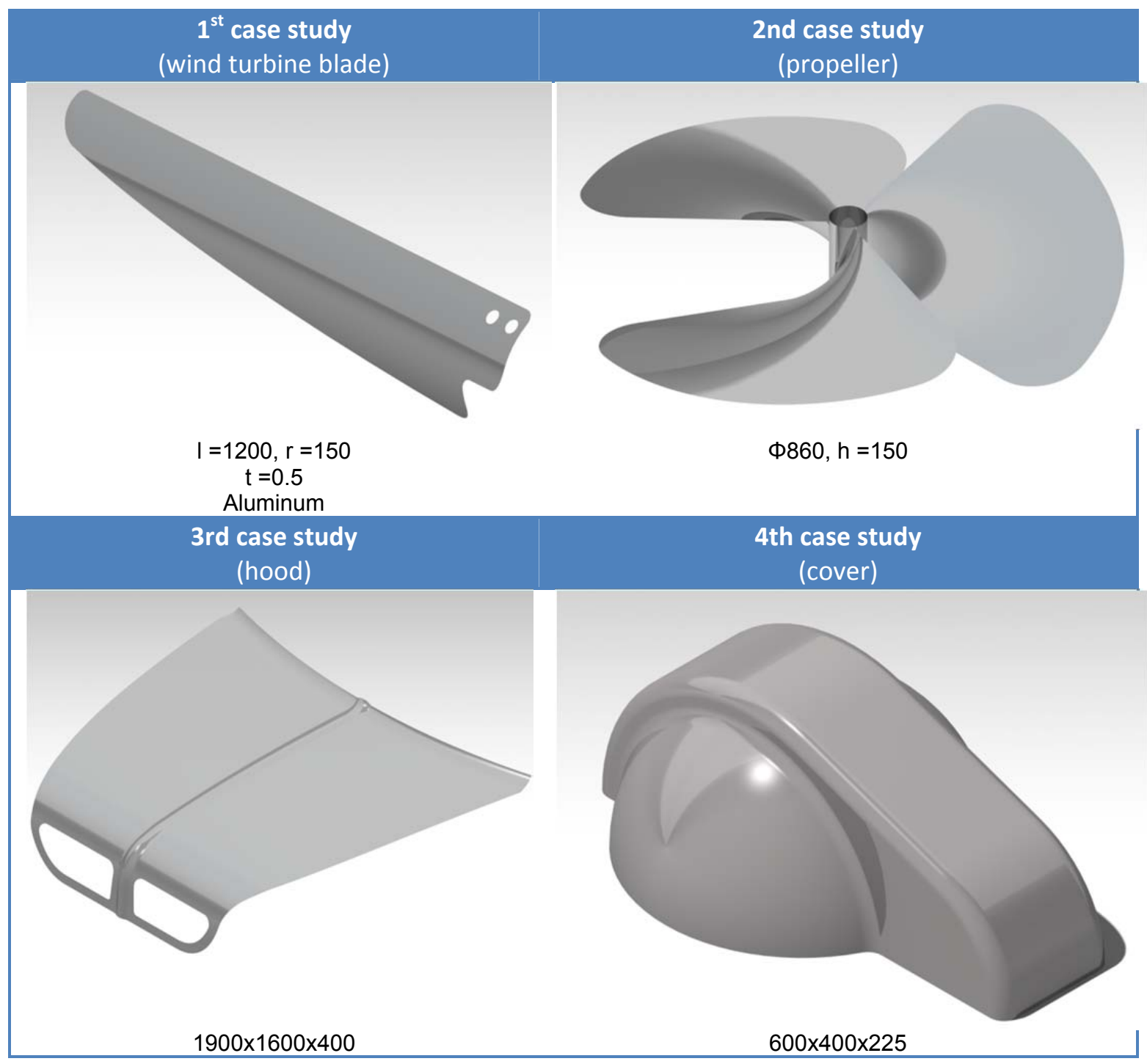

Note: Dimensions are in $\mathrm{mm}$. 


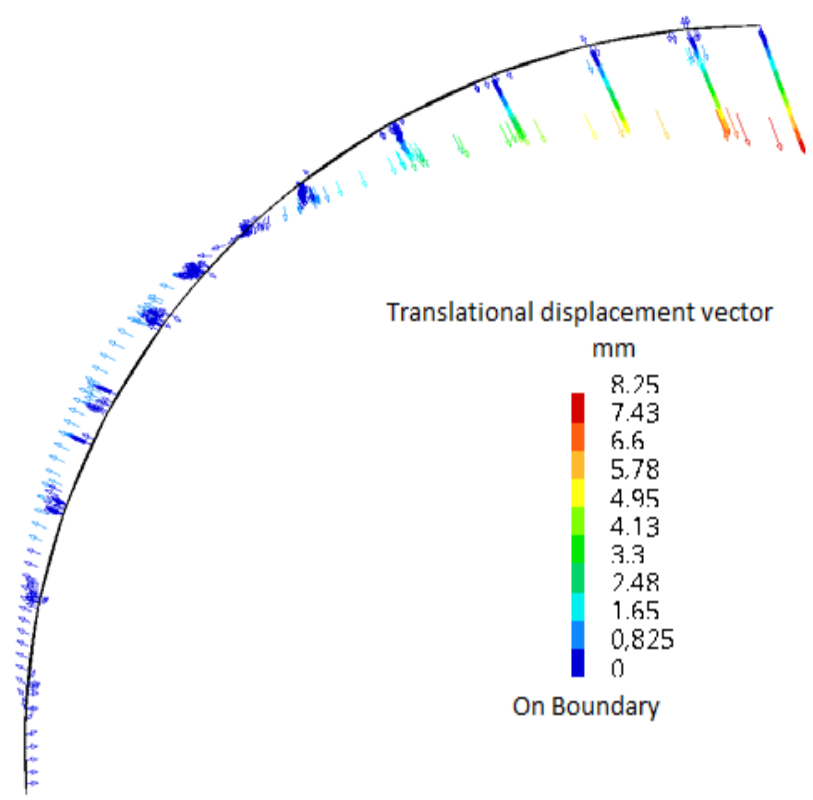

Figure 10: Predefined deformation for wind turbine blade (to simulate free-state) - Blade's side view

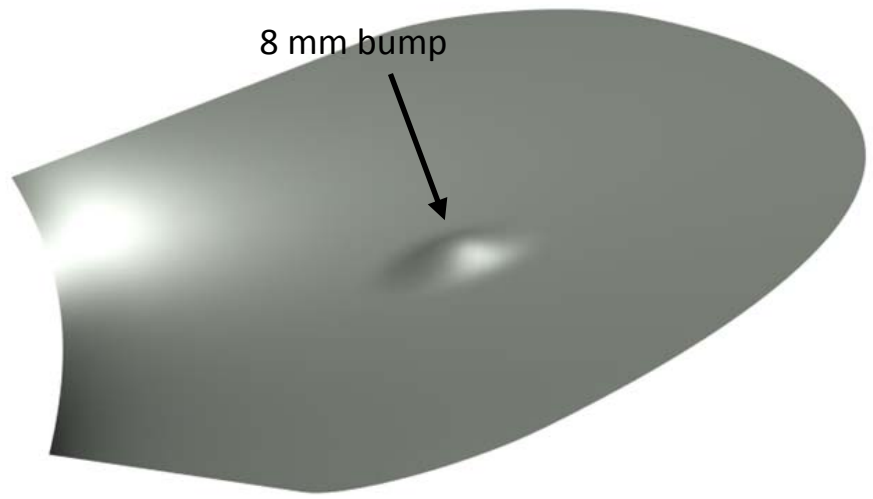

Figure 11: Sample predefined profile defect for $2^{\text {nd }}$ case study. 


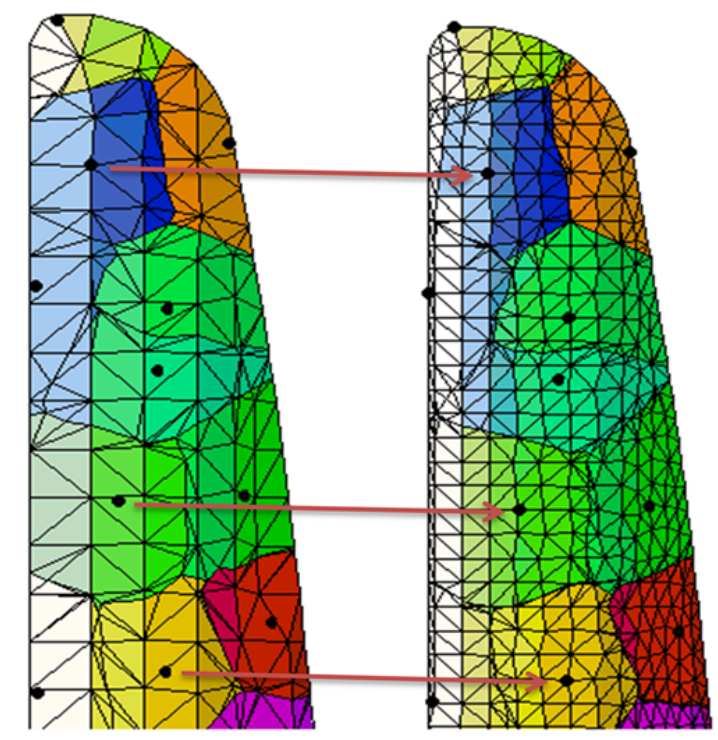

Figure 12: Similarity measure between CAD-model and range data (with 1833 and 7140 nodes respectively),

End of turbine blade ( ${ }^{\text {st }}$ case study)

Table 2: The verification of RNIF

\begin{tabular}{|c|c|c|c|c|c|c|c|c|}
\hline & \multicolumn{2}{|c|}{$1^{\text {st }}$ case study } & \multicolumn{2}{|c|}{$2^{\text {nd }}$ case study } & \multicolumn{2}{|c|}{$3^{\text {rd }}$ case study } & \multicolumn{2}{|c|}{$4^{\text {th }}$ case study } \\
\hline & GNIF & $R N I F$ & GNIF & $R N I F$ & GNIF & $R N I F$ & GNIF & $R N I F$ \\
\hline \multirow{6}{*}{ Results } & $2.67^{\mathrm{a}}$ & 2.93 & 4.44 & 4.51 & & & 2.09 & 2.16 \\
\hline & $(50)^{b}$ & (50) & (100) & $(100)$ & $x$ & $X$ & (100) & $(100)$ \\
\hline & 2.85 & 3.20 & 7.52 & 7.65 & & & 6.95 & 7.09 \\
\hline & (500) & (500) & (250) & $(250)$ & $x$ & $X$ & (500) & (500) \\
\hline & 4.57 & 4.71 & 7.87 & 7.83 & & & 9.19 & 9.38 \\
\hline & (1500) & $(1500)$ & $(500)$ & $(500)$ & $x$ & $x$ & $(1000)$ & (1000) \\
\hline $\begin{array}{l}\text { Maximum } \\
\text { predefined } \\
\text { profile } \\
\text { deviation }\end{array}$ & \multicolumn{2}{|c|}{5} & \multicolumn{2}{|c|}{8} & & & \multicolumn{2}{|c|}{10} \\
\hline
\end{tabular}

All case studies were performed on an AMD Phenom(tm) II X4 B95 Processor 3.00 GHz PC using a 64-bit operating system. For instance, similarity measure on first case study and for 50, 500 and 1500 sampled points took 1.2min, 9.9min and 117.8min, respectively. Correspondence search and putting the results with nonrigid registration algorithm are the main computational demanding steps. As expected, increasing the density of sampled points causes more accurate results (see case studies by column in 
Table 2). On one hand, in order to better represent the underlying surface deviation, we prefer the point sampling to be as densely as possible. On the other hand, we need to keep in mind that the discrete representation is used by computer algorithms, and every additional point increases storage and computational complexity costs.

\section{Conclusion}

As a result of the lack of theoretical knowledge in nonrigid geometric inspection, today in industry the only method to handle this problem is to use the high cost inspection fixtures. In this study we tried to construct the robust foundation to handle the fixtureless nonrigid inspection. Including part compliance with intrinsic geometry of surface in metrology of free-form surfaces is an area of research pioneered with this research. We introduced a methodology based on the fact that the shortest path (geodesic distance) between any two points on a shape remains unchanged during an isometric deformation. We call this property as distance preserving property of nonrigid parts. We merged the technologies in metric and computational geometry along with nonlinear dimensionality reduction methods and finite element analysis to develop a general approach to the geometrical inspection of nonrigid parts. The preliminary results proved that proposed methods, based on distance preserving NLDR methods was quite efficient. Although we tried to present the persuasive results by applying the developed methodology into some typical virtual mechanical parts, no method with such promise is likely to be widely accepted until more real-world tests can be done. A real-world test would indeed have immense utility. This test needs inspection fixture in addition to the work-piece. The results of this test, then, can be compared with our results. Our next study is to develop and verify the proposed methodology with real-world tests in cooperation with our industrial partner. Also in this study we did not deal with the effect of the diverse smoothing methods to reduce the topological noise effect. The uncertainty associated with the material properties has not been considered. These methods as well as the effect of material uncertainty should also be studied in depth. 
The proposed method is not a perfect and faultless substitution for inspection fixtures and CMM reports. However, in real-time applications it can be used for variational control of production lines so there will be no more need to stop production to test a workpiece. Although there are few researches in geometric inspection of nonrigid parts, general-purpose, fully automated and real-world practical method which can be a substitute for CMM reports does not yet exist. Specific long-term goals must be set forth and systematically accomplished.

\section{Acknowledgement}

The authors would like to thank the National Sciences and Engineering Research Council (NSERC) for support and financial contribution. Also, we appreciate the authors of different codes in NLDR and GMDS methods.

\section{Nomenclature}

\begin{tabular}{|ll|}
\hline$y_{i}^{\prime}$ & image of $x_{i} \mid x_{i} \in X$ in $Y$ \\
$\sigma$ & stress (loss function) \\
$\left(X, d_{X}\right)$ & metric space where $d$ is a metric on $X$ \\
$a_{i j}$ & ijth element of matrix $A$ \\
$d i s f$ & distortion of the map $f$ \\
$D_{X}$ & symmetric matrix of pairwise geodesic distances. (For $n$ \\
& points, it requires $\frac{n(n-1)}{2}$ calculations) \\
$d_{X}(a, b)$ & distance between a pair of points \\
$\mathbb{R}^{m}$ & $m$-dimensional Euclidean space \\
$t_{i}$ & triangle index \\
$t$ & workpiece thickness \\
$\operatorname{tr}(V)$ & Trace of matrix $V$ \\
$V^{T}$ & Transpose of matrix $V$ \\
\hline
\end{tabular}




\begin{tabular}{|ll|}
\hline$X, Y$ & surface \\
$Y_{M}$ & space $Y$ sampled by $M$ points \\
\hline
\end{tabular}

\section{References}

[1] H. Radvar-Esfahlan and S. A. Tahan, "Nonrigid Geometric Metrology using Generalized Numerical Inspection Fixtures," Precision Engineering, 2011.

[2] P. Besl and H. McKay, "A method for registration of 3-D shapes," IEEE Transactions on pattern analysis and machine intelligence, vol. 14, pp. 239-256, 1992.

[3] J. Bentley, "Multidimensional binary search trees used for associative searching," Communications of the ACM, vol. 18, p. 517, 1975.

[4] M. Greenspan and G. Godin, "A nearest neighbor method for efficient ICP," 2001.

[5] S. Rusinkiewicz and M. Levoy, "Efficient variants of the ICP algorithm," 2001, pp. 145152.

[6] M. Holden, "A review of geometric transformations for nonrigid body registration," Medical Imaging, IEEE Transactions on, vol. 27, pp. 111-128, 2008.

[7] I. Borg and P. J. F. Groenen, Modern multidimensional scaling: Theory and applications: Springer Verlag, 2005.

[8] E. Schwartz, A. Shaw, and E. Wolfson, "A numerical solution to the generalized mapmaker's problem: flattening nonconvex polyhedral surfaces," IEEE Transactions on pattern analysis and machine intelligence, vol. 11, pp. 1005-1008, 1989.

[9] J. De Leeuw, "Applications of convex analysis to multidimensional scaling," 2005.

[10] A. Elad and R. Kimmel, "On bending invariant signatures for surfaces," IEEE Transactions on pattern analysis and machine intelligence, vol. 25, pp. 1285-1295, 2003.

[11] R. Kimmel and J. Sethian, "Computing geodesic paths on manifolds," Proc. Natl. Acad. Sci. USA, vol. 95, pp. 8431-8435, 1998.

[12] A. Bronstein, M. Bronstein, and R. Kimmel, "Generalized multidimensional scaling: a framework for isometry-invariant partial surface matching," Proceedings of the National Academy of Sciences, vol. 103, p. 1168, 2006.

[13] X. Chen and F. Schmitt, "Intrinsic surface properties from surface triangulation," 1992, pp. 739-743.

[14] M. Yang and E. Lee, "Segmentation of measured point data using a parametric quadric surface approximation," Computer-aided design, vol. 31, pp. 449-457, 1999.

[15] A. Alrashdan, S. Motavalli, and B. Fallahi, "Automatic segmentation of digitized data for reverse engineering applications," IIE transactions, vol. 32, pp. 59-69, 2000.

[16] D. Anguelov, B. Taskar, V. Chatalbashev, D. Koller, D. Gupta, G. Heitz, and A. Ng, "Discriminative learning of markov random fields for segmentation of 3d scan data," 2005.

[17] D. Munoz, N. Vandapel, and M. Hebert, "Directional associative markov network for 3-d point cloud classification," 2008.

[18] R. Shapovalov and A. Velizhev, "Cutting-Plane Training of Non-associative Markov Network for 3D Point Cloud Segmentation."

[19] F. Chen, G. M. Brown, and M. Song, "Overview of three-dimensional shape measurement using optical methods," Optical Engineering, vol. 39, p. 10, 2000. 
[20] E. Savio, L. De Chiffre, and R. Schmitt, "Metrology of freeform shaped parts," CIRP Annals-Manufacturing Technology, vol. 56, pp. 810-835, 2007.

[21] J. Gao, N. Gindy, and X. Chen, "An automated GD\&T inspection system based on noncontact 3D digitization," International Journal of Production Research, vol. 44, pp. 117134, 2006.

[22] Y. Li and P. Gu, "Free-form surface inspection techniques state of the art review," Computer-Aided Design, vol. 36, pp. 1395-1417, 2004.

[23] Y. Li and P. Gu, "Inspection of free-form shaped parts," Robotics and ComputerIntegrated Manufacturing, vol. 21, pp. 421-430, 2005.

[24] Q. Shi and N. Xi, "Automated data processing for a rapid 3D surface inspection system," IEEE International Conference on Robotics and Automation

pp. 3939-3944, 2008.

[25] S. Ravishankar, H. Dutt, and B. Gurumoorthy, "Automated inspection of aircraft parts using a modified ICP algorithm," The International Journal of Advanced Manufacturing Technology, vol. 46, pp. 227-236, 2010.

[26] A. Weckenmann and A. Gabbia, "Testing formed sheet metal part using fringe projection and evaluation by virtual distortion compensation," in The 5th International Workshop on Automatic Processing of Fringe Patterns, 2005.

[27] A. Weckenmann, J. Weickmann, and N. Petrovic, "Shortening of inspection processes by virtual reverse deformation," presented at the 4th International Conference and Exhibition on Design and Production of Machines and Dies/Molds, Cesme, Turkey, 2007.

[28] P. Bourdet and A. Clément, "Controlling a complex surface with a 3 axis measuring machine," Annals of the CIRP, vol. 25, pp. 359-361, 1976.

[29] C. Lartigue, F. Theibaut, P. Bourdet, and N. Anwar, "DIMENSIONAL METROLOGY OF FLEXIBLE PARTS: IDENTIFICATION OF GEOMETRICAL DEVIATIONS FROM OPTICAL MEASUREMENTS," Advanced mathematical \& computational tools in metrology VII, p. 196, 2006.

[30] G. Abenhaim, S. Tahan, A. Desrochers, and R. Maranzana, "A novel approach for the inspection of flexible parts without the use of special fixtures " ASME, Journal of manufacturing science and engineering, 2011.

[31] B. Amberg, S. Romdhani, and T. Vetter, "Optimal step nonrigid ICP algorithms for surface registration," 2007, pp. 1-8.

[32] E. Dijkstra, "A note on two problems in connexion with graphs," Numerische mathematik, vol. 1, pp. 269-271, 1959.

[33] J. Sethian, "A fast marching level set method for monotonically advancing fronts," Proceedings of the National Academy of Sciences, vol. 93, p. 1591, 1996.

[34] A. Bronstein, M. Bronstein, M. Bronstein, and R. Kimmel, Numerical geometry of nonrigid shapes: Springer-Verlag New York Inc, 2007.

[35] D. Burago, Y. Burago, and S. Ivanov, A course in metric geometry, 2001.

[36] H. RADVAR-ESFAHLAN and S. A. TAHAN, "Distance preserving dimensionality reduction methods and their applications in geometric inspection of nonrigid parts," 5th SASTECH Conference, Iran., 2011.

[37] A. Bronstein, M. Bronstein, and R. Kimmel, "Efficient computation of isometry-invariant distances between surfaces," SIAM Journal on Scientific Computing, vol. 28, pp. 18121836, 2007. 